Artigo de revisão

\title{
VITEX AGNUS-CASTUS L., OENOTHERA BIENNIS L., CURCUMA LONGA L. COMO TRATAMENTO ALTERNATIVO NA SÍNDROME DA TENSÃO PRÉ-MENSTRUAL (TPM)
}

\author{
Autores: Karin Alessandra Honorato ${ }^{1, A}$, Rogério da Silva Veiga ${ }^{2}$ \\ ${ }^{1}$ Nutricionista, especialista em nutrição clínica funcional e nutrição esportiva funcional. \\ ${ }^{2}$ Farmacêutico-bioquímico com ênfase em alimentos, mestre em farmacologia, doutor em ciências da saúde.
}

\section{Informações do artigo}

Palavras Chave:

tensão pré-menstrual; fitoterapia; terapia alternativa.

\begin{abstract}
Resumo
A Síndrome da Tensão Pré-Menstrual (TPM) é um conjunto de sinais e sintomas que ocorrem ciclicamente relacionados com a menstruação, apresentando intensidade suficiente para interferir na vida social e profissional de muitas mulheres. Com base nisso, a popularidade das terapias complementares/alternativas tem crescido nos últimos anos. A fitoterapia tem sido umas dessas terapias alternativas que busca minimizar os efeitos dos sintomas da TPM e tem sido o tratamento preferido das mulheres por reduzirem os efeitos colaterais se comparados aos tratamentos farmacológicos. O presente estudo tem como objetivo rever a literatura sobre o uso de fitoterápicos na TPM como recurso terapêutico no alívio dos sintomas físicos e psicológicos. O levantamento bibliográfico foi conduzido a partir de artigos publicados sobre o assunto nos bancos de dados online. Em geral, estudos mostraram que Vitex agnus-castus L., Oenothera biennis L. e Curcuma longa L. atuam, por meios diferentes, no alivio aos sintomas da TPM. Assim, verificou-se que a fitoterapia é um método eficaz e seguro para o tratamento, alivio e melhora da qualidade de vida das mulheres que sofrem de TPM.

Palavras-chave: tensão pré-menstrual; fitoterapia; terapia alternativa.
\end{abstract}

\section{Abstract}

Premenstrual Tension Syndrome (PMTS) is a set of signs and symptoms that occur cyclically related to menstruation, presenting enough intensity to interfere in the social and professional life of many women. Based on

Keywords:

premenstrual tension syndrome; phytotherapy; alternative therapy.

\footnotetext{
${ }^{A}$ Autor correspondente:

Karin Als. Honorato: e-mail: karin@clinicatrinutrix.com.br - https://orcid.org/0000-0002-6800-9832
}

DOI: https://doi.org/10.31415/bjns.v2i2.54 - Artigo recebido em: 03 de abril de 2019; aceito em 22 de abril de 2019 ; publicado em 29 de maio de 2019. Brazilian Journal of Natural Sciences, Vol. 2, N.2, maio 2019. Disponível online a partir de 29 de maio de 2019, ISSN 2595-0584. www.bjns.com.br - Todos os autores contribuíram igualmente com o artigo. Este é um artigo de acesso aberto sob a licença CC - BY: http://creativecommons.org/licenses/by/4.0 
this, the popularity of complementary / alternative therapies has grown in recent years. Phytotherapy has been one of those alternative therapies that seeks to minimize the effects of PMTS symptoms and has been the preferred treatment of women for reducing side effects compared to pharmacological treatments. The present study aims to review the literature on the use of herbal medicines in PMTS as a therapeutic resource in the relief of physical and psychological symptoms. The bibliographic survey was conducted from articles published on the subject in online databases. In general, studies have shown that Vitex agnus-castus L., Oenothera biennis L. and Curcuma longa L. act by different means to relieve symptoms of PMTS. Thus, it has been found that phytotherapy is an effective and safe method for the treatment, relief and improvement of the quality of life of women suffering from PMTS.

\section{Introdução}

O uso de plantas medicinais no processo de prevenção e tratamento de diversas doenças é uma prática que acompanha a humanidade desde o seu começo (OLIVEIRA et. al., 2017). As propriedades terapêuticas de determinadas plantas foram descobertas e propagadas de geração a geração resultando na grande disseminação do uso das mesmas (SANTOS, LOPES, 2015; OLIVEIRA et. al., 2017). Com base nisso, a fitoterapia é o método que baseia a cura ou prevenção de doenças a partir do uso do principio ativo extraído de plantas medicinais em associação com os cuidados necessários (OLIVEIRA et. al., 2017).

As oportunidades para a identificação de produtos com possível utilização farmacêutica aumentam com a diversidade das espécies. As plantas constituem um verdadeiro tesouro verde porque carregam os denominados princípios ativos, preparados pela engenharia química da natureza em milhões de anos de eventos. O controle e o conhecimento desse tesouro são hoje motivo de grande importância tanto para os curandeiros quanto para os grandes laboratórios farmacêuticos (SANTOS, LOPES, 2015).

Atualmente há um crescimento na busca e na intensificação do uso de plantas pela população, com fins medicinais. Em consequência disso, um crescimento também da fitoterapia (SANTOS, LOPES, 2015). Ela tem se tornado um método de tratamento alternativo comum para muitas doenças que além de mais econômico é também um método com menos efeitos colaterais do que os fármacos (MALEKI-SAGHOONI et. al., 2018; DELARAM, 2014).

Os fitoterápicos têm sido relatados como método eficaz no tratamento da síndrome da tensão pré- menstrual (DELARAM, 2014). Apesar dos muitos tratamentos farmacológicos, muitas mulheres preferem abordagens não farmacêuticas incluindo mudanças na dieta, exercícios, terapia cognitiva comportamental e a medicina alternativa (JANG, KIM, CHOI, 2014). A tensão pré-menstrual (TPM) é um dos problemas mais comuns entre as mulheres em idade reprodutiva e é considerada um transtorno biológico psico-endócrino (DELARAM, 2014). A TPM referese a um conjunto de sintomas psicológicos e físicos (MALEKI-SAGHOONI et. al., 2018) que comprometem a vida social, profissional e familiar das mulheres acometidas por ela (SANTOS, LOPES, 2015).

Neste contexto, este trabalho tem como objetivo realizar uma investigação sistemática dos fitoterápicos que fazem parte do arsenal terapêutico utilizado para o tratamento da tensão pré-menstrual.

\section{Material e método}

Esta pesquisa foi realizada através de revisão bibliográfica de artigos publicados no período de 2013 a 2018, disponíveis nas bases de dados Pubmed, Periodicos Capes, Google acadêmico utilizando-se os termos em inglês para busca de palavras chaves: "Phytotherapy", "phytotherapy and premenstrual syndrome", "premenstrual syndrome", "Agnus castus and premenstrual syndrome", "Agnus castus", "primrose oil", "primrose oil and premenstrual syndrome", "Oenothera biennis L., "Curcuma longa", "Curcuma longa and premenstrual syndrome","Curcumin.

\section{Discussão}

\section{Síndrome da Tensão Pré-Menstrual}


A Síndrome Pré-Menstrual (SPM), também conhecida como tensão pré-menstrual (TPM) é um transtorno biológico psico-endócrino (DELARAM, 2014) que atinge muitas mulheres, tornando-se um incômodo para a maioria delas em sua fase reprodutiva. A etiologia desse transtorno é multifatorial. Causas precisas e a influência da hereditariedade ainda são desconhecidas e ainda não suficientemente exploradas. Algumas pesquisas sobre as causas da TPM mostram complexos mecanismos envolvendo hormônios ovarianos, opióides endógenos, neurotransmissores, prostaglandinas, sistema nervoso autônomo, sistema endócrino, entre outros (SANTOS, LOPES, 2015).

Os sintomas da TPM, que podem ser psicológicos e físicos, surgem entre 10 a 14 dias antes da menstruação, desaparecendo no inicio do fluxo menstrual (SANTOS, LOPES, 2015). Até $25 \%$ das mulheres relatam sintomas de moderados a graves. Aproximadamente 5\% relatam sintomas graves (JANG, KIM, CHOI, 2014). Os sintomas psicológicos incluem ansiedade, depressão, irritabilidade, nervosismo, choro, falta de concentração, agressão e tendências suicidas. Alguns dos sintomas físicos são dores de cabeça, inchaço abdominal, sensibilidade nos seios, dores musculares e articulações, fadiga e mudança de apetite (DELARAM, 2014).

Os tratamentos convencionais, como intervenções hormonais e antidepressivos sintéticos, não são as opções preferidas para várias mulheres. Sendo assim, as mulheres recorrem frequentemente a medicamentos complementares para estas e outras preocupações relacionadas com a saúde reprodutiva (VAN DIE, et. al., 2013). Tratamentos não farmacológicos incluem mudança nos hábitos alimentares, redução do consumo de sal e gordura animal, exercício, redução do estresse (DELARAM, 2014), além do tratamento alternativo que inclui a fitoterapia.

Muitas pesquisas têm sido realizadas para investigar os efeitos dos fitoterápicos para o tratamento da TMP (MALEKI-SAGHOONI et. al., 2018; SANTOS, LOPES, 2015; JANG, KIM, CHOI, 2014; DELARAM, 2014; SAKI et. al., 2014).

\section{O uso de fitoterápicos na Síndrome da Tensão Pré-Menstrual (TPM)}

\section{Vitex agnus-castus $\mathrm{L}$.}

Vitex agnus-castus L. (Lamiaceae), é originalmente nativa do Mediterrâneo, sendo popularmente conhecida como agno-casto, árvore-da-castidade, alecrimde-angola, cordeiro-casto, flor-da-castidade. A parte utilizada são as folhas e os frutos, que se utiliza popularmente na forma de chá para o tratamento da TPM, menopausa e como anafrodisíaco (SANTOS, LOPES, 2015). Além disso, é empregado para uma variedade de outros distúrbios reprodutivos femininos (RAFIEIAN-KOPAEI, MOVAHEDI, 2017; VAN DIE, et. al., 2013), como para aliviar a menstruação, o controle do sangramento de miomas uterinos, síndrome do ovário policístico e mulheres com enxaqueca (VAN DIE, et. al., 2013).

A Associação de Profissionais da Saúde Reprodutiva recomenda $V$. agnus-castus L. para o tratamento da TPM (ASSOCIATION OF REPRODUCTIVE HEALTH PROFESSIONALS, 2018). Seu extrato tem se mostrado eficaz, seguro e bem tolerado em mulheres com síndrome pré-menstrual (RAFIEIAN-KOPAEI, MOVAHEDI, 2017). Essa eficácia é fortemente apoiada por evidências de múltiplos ensaios clínicos (HESKES et. al., 2018). Seus princípios ativos são óleos essenciais, flavonoides e glicosídeos iridoides (SANTOS, LOPES, 2015). As propriedades farmacológicas de $V$. agnus-castus L. são atribuídas à presença de ligantes de dopamina, opióides e receptores de estrogênio que estão envolvidos na modulação dos níveis hormonais que afetam a TPM (HESKES et. al., 2018).

$\mathrm{O}$ efeito benéfico dos extratos de $V$. agnus-castus L. no tratamento de sintomas da TPM causou um crescente interesse na determinação de seus possíveis mecanismos de ação nos sintomas da TPM (KHALILZADEH et. al., 2015), porém esses mecanismos ainda não são bem elucidados (VERKAIK et. al., 2017).

Recentemente, Webster et. al., (2011) relataram que os efeitos terapêuticos de diferentes frações do extrato de $V$. agnus-castus L. são mediados através da ativação de receptores opióides. Outros estudos in vitro mostram a ligação de extratos $V$. agnus-castus L. ao receptor de dopamina-2, resultando em inibição da prolactina, ao receptor opióide humano e uma afinidade de ligação seletiva para o receptor de $\beta$-estrogênio (VERKAIK et. al., 2017). Em um estudo na Alemanha, foram investigados os casos de TPM em que o extrato vegetal de V. agnus-castus L. foi utilizado como método complementar. Outro estudo avaliou a eficácia $V$. agnus-castus L. na TPM. Os resultados mostraram que a planta é muito benéfica no tratamento de TPM moderada a grave (RAFIEIAN-KO- 
PAEI, MOVAHEDI, 2017).

Hoje, o V. agnus-castus L. está disponível em uma variedade de formas farmacêuticas, incluindo tinturas, extratos líquidos, comprimidos e preparações homeopáticas, e é comumente usado em todo o mundo (VAN DIE, et. al., 2013).

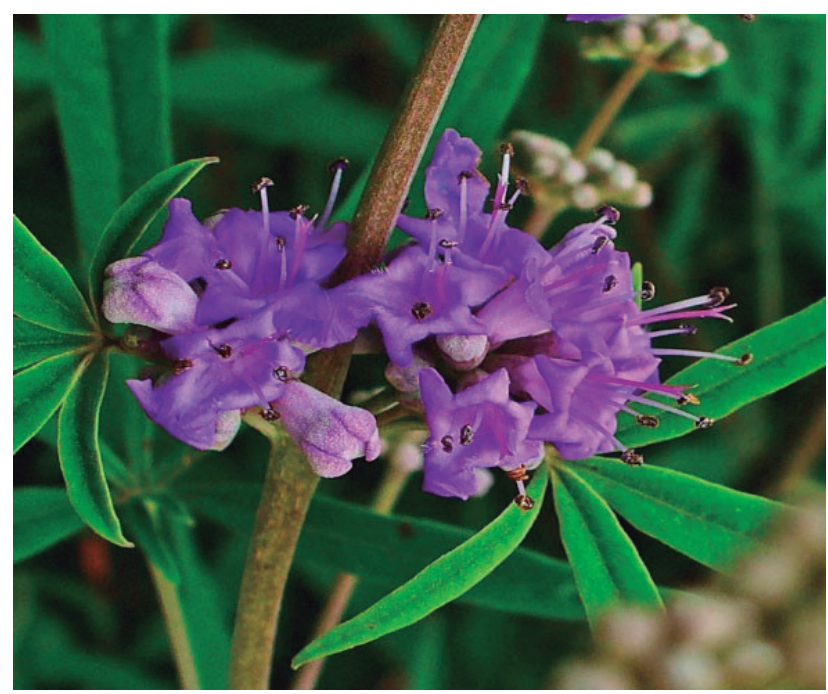

Flores de Vitex agnus-castus L., popularmente conhecida como agno-casto, árvore-da-castidade, alecrimde-angola, cordeiro-casto, flor-da-castidade. Fonte: Página do Safari Garden ${ }^{1}$.

${ }^{1}$ (Disponível em: <https://www.safarigarden.com.br/ muda-de-vitex-agnus-castus $>$ Acesso em: dez. 2018).

\section{Oenothera biennis L. (Óleo de prímula)}

A prímula (Oenothera biennis L.) é uma planta pertencente à família Onagraceae e o seu óleo é obtido a partir das sementes (TIMOSZUK, BIELAWSKA, SKRZYDLEWSKA, 2018).

A planta é nativa da América do Norte e foi introduzida na Europa como planta ornamental (SANTOS, LOPES, 2015).

As sementes contêm dois ácidos graxos essenciais: ácido linoleico (LA) e ácido gama-linoleico (GLA). O ácido gama-linoleico (GLA) é um dos ácidos graxos raros que são encontrados em algumas espécies de plantas. A prímula é considerada a principal fonte de GLA (SAKI et. al., 2014). O ácido linoleico e o ácido gama-linoleico contribuem para o bom funcionamento de muitos tecidos do corpo humano, pois são precursores de compostos que levam à geração de eicosanóides anti-inflamatórios como, por exemplo, as prostaglandinas E1 (TIMOSZUK, BIELAWSKA,
SKRZYDLEWSKA, 2018).

Existem evidências que comprovam a eficiência do óleo de prímula na tensão pré-menstrual (TPM) (SAKI et. al., 2014), pois se presume que a ingestão de GLA contido no óleo de prímula cause uma resposta anti-inflamatória, sendo uma consequência do aumento da síntese da prostaglandina E1 (SAKI et. al., 2014; CHUNG et. al., 2018). As prostaglandinas de série E1 exercem efeito regulador dos hormônios sexuais femininos, estrógenos, progesterona e prolactina (SANTOS, LOPES, 2015).

Os principais sintomas da TPM como irritabilidade, dores de cabeça, inchaço de seios, dores e inchaços abdominais, edema e ganho de peso estão relacionados a alterações no metabolismo de prostaglandinas. Portanto, a ingestão de alimentos ricos em GLA, como o óleo de prímula, auxilia na regulação do metabolismo das prostaglandinas, atenuando os sintomas da tensão pré-menstrual (SANTOS, LOPES, 2015).

O óleo das sementes de prímula é utilizado em mais de 30 países como um suplemento nutricional, contribuindo para uma boa qualidade de vida (SANTOS, LOPES, 2015).

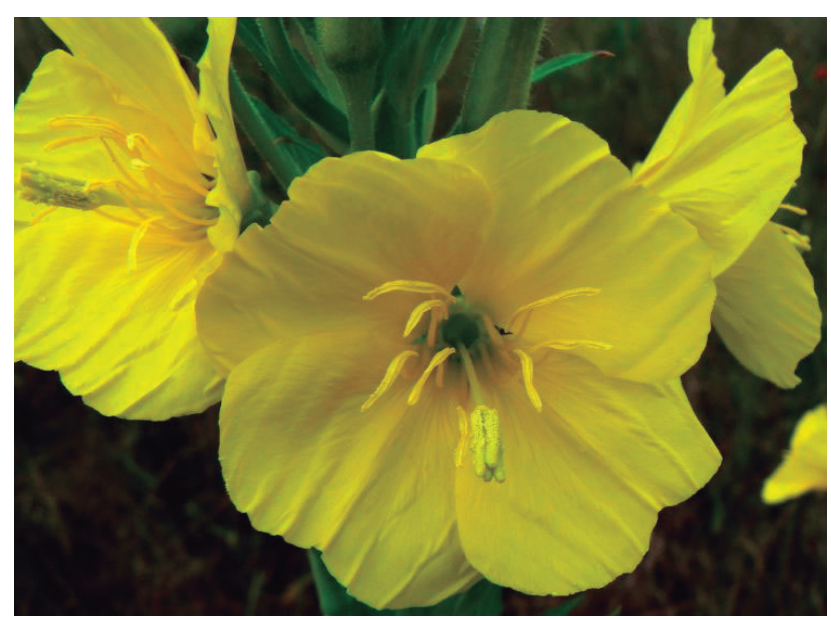

Flores de Oenothera biennis L., popularmente conhecida como prímula. Fonte: Biolib.cz. ${ }^{2}$

${ }^{2}$ (Disponível em: <https://www.biolib.cz/en/image/ id74692/> Acesso em: dez. 2018).

\section{Curcuma longa L. (Cúrcuma)}

A Curcuma longa L. é uma planta nativa da Ásia, pertencente à família Zingiberaceae e com o nome comum de açafrão (MOGHADAMTOUSI et. al., 2014). A curcumina é o extrato e composto ativo derivado do rizoma da cúrcuma que apresenta cor amarela e é 
usada na forma de pó como agente de coloração e sabor na indústria alimentícia e na fitoterapia em países asiáticos há milhares de anos para tratar vômitos, dor de cabeça, diarreia, etc (KHALAJI et. al., 2018; LIU et. al., 2018).

Recentemente, estudos farmacológicos revelaram que a curcumina tem fortes atividades antioxidantes, antimicrobianas, anti-inflamatórias e anticancerígenas. Evidências crescentes mostram que a curcumina é um produto muito seguro para o ser humano (LIU et. al., 2018).

É usado extensivamente como um remédio caseiro para várias doenças, incluindo distúrbios menstruais (GANGOLLI et. al., 2015). Um estudo revelou que a curcumina aumenta os níveis de prolactina em mulheres com tensão pré-menstrual (KHALAJI et. al., 2018). Além disso, atua como um antiespasmódico reduzindo as cólicas menstruais, também ajuda a regular as atividades do sistema reprodutor feminino, ajuda a equilibrar os níveis hormonais e a purificar o útero através da remoção do sangue residual na forma de menstruação, tratando assim os períodos irregulares (GANGOLLI et. al., 2015).

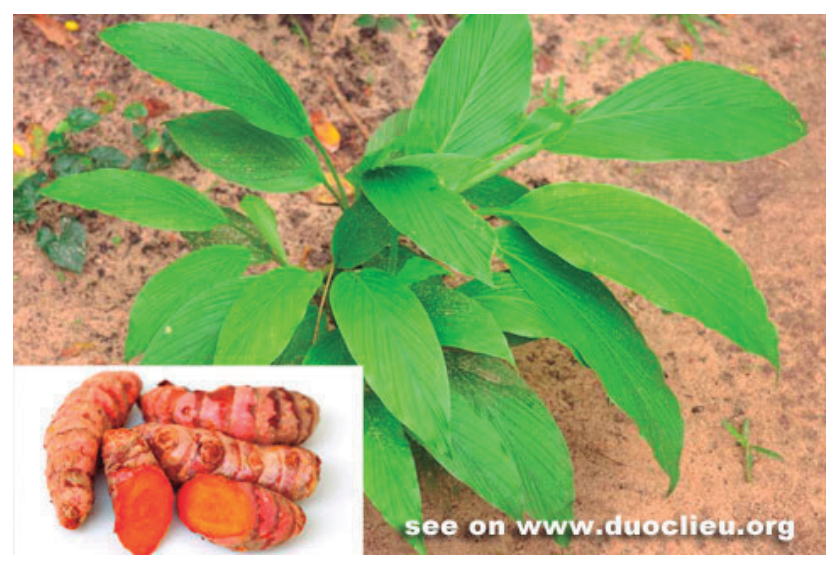

Rizoma Curcuma longa L., popularmente conhecida como cúrcuma, açafrão. Fonte: Pharmacognosy. ${ }^{3}$

${ }^{3}$ (Disponível em: <http://www.epharmacognosy. com/2012/04/curcuma-root-yujin-curcuma-longa-l. html> Acesso em: dez. 2018).

\section{Conclusão}

A TPM é uma síndrome comum em mulheres na fase reprodutiva com sintomas psicológicos e físicos que podem interferir na vida profissional, social e familiar. Esses sintomas podem ser amenizados com tratamentos, não somente com os medicamentos sintéticos e isolados, mas também com tratamentos alternativos. Os tratamentos alternativos têm sido os preferidos e mais procurados entre as mulheres que sofrem desses sintomas. Diante disso, este estudo reforça a importância dos fitoterápicos na abordagem farmacológica desta síndrome.

Os sintomas e a intensidade podem variar muito de mulher pra mulher e por isso não existe um tratamento especifico para a TPM. Assim, os fitoterápicos assumem relevância com um crescimento em escala mundial do uso de diferentes extratos vegetais.

Os medicamentos fitoterápicos utilizados na TPM apresentam segurança e eficácia no alívio da menstruação, dores de cabeça, melhora dos sintomas da irritabilidade, alívio das dores provocadas por cólicas e inchaços. No entanto, fitoterápicos não devem ser utilizados abusivamente e sem orientação de um profissional da saúde.

\section{Referências}

Association of Reproductive Health Professionals. A quick reference guide for clinicians. Managing premenstrual symptoms. [acesso em $2018 \mathrm{dez} 7$ ]. Disponível em: https://www.arhp.org/31-publications-and-resources/quick-reference-guide-for-clinicians/ managing-premenstrual-symptoms

CHUNG B.Y, Parque SY, Jung MJ, Kim HO, Parque CW. Effect of Evening Primrose Oil on Korean Patients With Mild Atopic Dermatitis: A Randomized, Double-Blinded, Placebo-Controlled Clinical Study. Ann Dermatol. 2018 Ago; 30(4): 409-416.

DELARAM, M. Treatment of Moderate to Severe of Premenstrual Syndrome with Echinophora platyloba. ZJRMS. 2014; 16(3): 50-54.

GANGOLLI, D.; Rane R.; Chotalia C.; Kundalwal S.; Salkar K.; Suthar, A. Qualitative \& quantitative phytochemical assessment of \#PPL/2013 capsules - A poly-herbal formulation for gynecological disorders. The Pharma Innovation Journal. 2015; 4(2): 77-82.

HESKES, A.M.; Sundram, T.C.M.; Boughton, B.A.; Jensen, N.B.; Hansen, N.; Crocoll, C.; Biosynthesis of bioactive diterpenoids in the medicinal plant Vitex agnus-castus. Plant J. 2018 Mar; 93(5): 943-958.

JANG, S.H.; Kim, D.; Choi, M.S. Effects and treatment methods of acupuncture and herbal medicine for premenstrual syndrome/pré-menstrual dysphoric disorder: systematic review. BMC Complementary and Alternative Medicine. 2014 Jan 10; 14(11). 
KHALAJI, N.; Namyari, M.; Rasmi, Y.; Pourjabali, M.; Chodari, L. Protective effect of curcumin on fertility of rats after exposure to compact fluorescent lamps: An experimental study. Int. J. Reprod. Biomed. 2018 Jul; 16(7): 447-454.

KHALILZADEH, E.; Vafaei, Saiah L.; Hasannejad, H.; Ghaderi, A.; Ghaderi, S.; Hamidian L. Antinociceptive effects, acute toxicity, and chemical composition of Vitex agnus-castus essential oil. Avicenna J. Phytomed. 2015 May/Jun; 5(3): 218-230.

LIU, Z.; Huang, P.; Siukan, L.; Tian, H.; Leung, W.; $\mathrm{Xu}, \mathrm{C}$. Preventive Effect of Curcumin Against Chemotherapy-Induced Side-Effects. Front. Pharmacol. 2018 Nov 27; 9: 1374.

MALEKI, S. N.; Karimi, F.Z.; Behboodi Moghadam Z.; Mirzaii Najmabadi K. The Effectiveness and Safety of Iranian Herbal Medicines for Treatment of Premenstrual Syndrome: A Systematic Review. Avicenna J. Phytomed. 2018 Mar/Abr; 8(2): 96-113.

MOGHADAMTOUSI, S.Z.; Kadir, H.A.; Hassandarvish, P.; Tadjique, H.; Abubakar, S.; Zandi K. A. Review on Antibacterial, Antiviral, and Antifungal Activity of Curcumin. Biomed Res. Int. 2014 Abr 29; 2014.

OLIVEIRA, A.F.P.; Costa, I.C.P.; Andrade, C.G, Santos KFO, Anízio BKF, Brito FM. Phytotherapy .; Primary care: Study with nurse professionals. Rev. Fund. Care Online. 2017 Abr/Jun; 9(2): 480-487.

RAFIEIAN, Kopaei M.; Movahedi, M. Systematic Review of Premenstrual, Postmenstrual and Infertility Disorders of Vitex Agnus Castus. Electronic Physician. 2017 Jan 25; 9(1): 3685-3689.

SAKI. M.; Akbari, S.; Saki, M.; Tarrahi, M.J.; Gholami, M.; Pirdadeh , S. The effect of primrose oil on the premenstrual syndrome among the female students in Lorestan University of Medical Sciences: A triple blind study. JNMS. 2015; 2(1): 20-26.

SANTOS, T.D.; Lopes, G.C. Premenstrual syndrome (PMS): phytotherapy evidence based. Rev. Uningá Review. 2015 Nov 14; 24(3): 139-145.

TIMOSZUK, M.; Bielawska, K.; Skrzydlewska, E. Evening Primrose (Oenothera biennis) Biological Activity Dependent on Chemical Composition. Antioxidants. 2018 Ago 14; 7(8): 108.

Van Die M.D, Burger, H.G.; Teede, H.J.; Bone, K.M. Vitex agnus-castus Extracts for Female Reproductive Disorders: A Systematic Review of Clinical Trials. Planta Med. 2013 Mai; 79(7): 562-575.

VERKAIK, S.; Kampermam, A.M.; Van Westrhe- nen R.; Schulte, P.F.J. The treatment of premenstrual syndrome with preparations of Vitex agnus castus: A systematic review and meta-analysis. Am. J. of Obstet. and Gynecol. 2017; 217(2): 150-166.

WEBSTER, D.E.; He Y.; Chen, S..; Pauli, G..; Farnsworth, N.R.; Wang, Z.J. Opioidergic mechanisms underlying the actions of Vitex agnus-castus L. Biochem pharmacol. 2011 Jan 1; 81(1): 170-177. 